\title{
Privacy and Personal Identity
}

\author{
Wade L. Robison \\ Ezra A. Hale Professor in Applied Ethics \\ Rochester Institute of Technology
}

\begin{abstract}
What marks the traditional privacy torts of disclosure, intrusion, false light, and appropriation is that they require an invasion, an intrinsic harm caused by someone doing something to us without our consent. But we are now voluntarily giving up information about ourselves-to our physicians, for instance-that is being gathered into databases that are' bought and sold and that can be appropriated by those who wish to assume our identities. The way in which our privacy is put at risk is different, and this leads to a new understanding of the concept of privacy. Others appropriate our identities, treating us as objects; by doing so, our standing as autonomous moral agents, controlling how we present ourselves to the world, is thus denied.
\end{abstract}

Key words: privacy, personal identity

We are all familiar with having our privacy invaded - by someone reading our diary, spreading a secret, coming into where we thought we would be alone, or listening in on a conversation without our knowledge. The use of new technologies only seems to provide simple extensions of the ways in which our privacy has been traditionally invaded. What difference does it make whether others open envelopes to read our mail or break into our computer accounts to look at our e-mail? Either way they are obtaining information about us that we did not give them permission to obtain, information that we thought was private. Technology has certainly created new ways in which others can invade our privacy-by reading our e-mail, using high-powered listening devices to hear through our walls, recording our telephone transmissions (as Princess Di, Prince Charles, and Newt Gingrich have discovered). So the mode of entry is different. Yet the kind of harm seems the same.

If we have these sorts of examples in mind, we may well think that though new technologies will produce increased concerns about how much and how often our privacy can be invaded, it will do nothing to change our concept of privacy. The concept will remain the same although the ways in which our privacy can be invaded

Requests for reprints should be sent to Wade L. Robison, College of Liberal Arts, Rochester Institute of Technology, 92 Lomb Memorial Drive, Rochester, NY 14623-5604. 
change. Life after computers and other technologies may seem like life after the invention of modern forms of transportation. It used to be that a pedestrian could be run down by a horse or a carriage or a cart. Now a pedestrian can be run down by a car or a truck or a motorcycle or a bike or a bus or a train. A pedestrian's life is more dangerous because there are more ways in which one can be run down, by bigger things, going much faster. But, still, one is being run down. The extent and magnitude of harms may differ, but the kind of harm remains the same.

Recent popular writing on privacy suggests just this view about the impact of new technologies on the concept of privacy. For instance, in The Right to Privacy (Alderman \& Kennedy, 1995), the cases include a couple being videotaped having sex, the tape then being played at various gatherings (Cooper $v$. Anderson), a television crew in vading a home to videotape a death (Miller v. NBC), and managers examining e-mail (Shoars v. Epson America, Inc.; Alderman \& Kennedy, 1995, pp. $158 \mathrm{ff}$., $227 \mathrm{ff}$., $310 \mathrm{ff}$.). Someone walking in on you uninvited while you are having sex is a harm, but nowhere near as extensive a harm as someone secretly videotaping your having sex to play for others. Someone reading a letter of yours without permission is a harm, but nowhere near as extensive a harm as someone having access to all your e-mail. But though the scope of the harms is greater, they are not different in kind. Technological advances only make it easier, Alderman and Kennedy must think, to invade your privacy in the old-fashioned ways.

Indeed, Alderman and Kennedy's (1995) book is organized around the privacy torts that have developed in the law since 1890 , when Warren first became upset at what he thought was an invasion of privacy and proceeded to write, with Brandeis, an article for the Harvard Law Review arguing for a right to privacy (Warren \& Brandeis, 1890), and 1902, when the first case involving a privacy righ was decided (Prosser, 1960, p. 385). Prosser has said of the torts that have developed that they involve

four distinct kinds of invasion of four different interests of the plaintiff, which are tied together by the common name, but otherwise have almost nothing in common except that each represents an interference with the right of the plaintiff ... "to be let alone." (p. 389)

He described the four harms in this way:

Intrusion upon the plaintiff's seclusion or solitude, or into his private affairs.

2. Public disclosure of embarrassing private facts about the plaintiff.

3. Publicity that places the plaintiff in a false light in the public eye.

4. Appropriation, for the defendant's advantage, of the plaintiff's name or likeness. (p. 389)

These are the privacy torts of intrusion, disclosure, false light, and appropriation, and it is examples of the first two that seem to come to mind most easily and suggest that privacy is something we have, as an odd sort of object, rather like an envelope that surrounds us-like the air we carry in with us when the weather is bitter cold. Others must come into contact or penetrate that envelope in some way for harm to occur. The person whose envelope of privacy is penetrated is essentially passive; the person who does the penetrating is essentially active-though we can readily imagine an invasion occurring through a failure to act, someone omitting to hang up the phone, for instance, when the person the call is for comes on the line.

In this image, an invasion of privacy occurs when others, through their acts or omissions, penetrate the envelope' of your privacy-either by becoming privy to information about you that you had not meant for them to see or by confronting you in some way in a place or situation that you thought would exclude them, at least at the time (as in a toilet stall). Looking at your e-mail, on this conception, is no different in kind than looking at your mail.

Things may seem significantly different because the extent and magnitude of the harm can be significantly greater. The person invading your e-mail can read more of what you have written, to more individuals, than by reading a single letter, and someone can, with listening devices, pick up conversations that, in 1890 , you would have expected to be private. And, of course, it is harder to protect yourself against such invasions. It is one thing to whisper a secret in public. It is quite another to figure out how to prevent those outside your house from hearing what you are saying when they can use ultra-sensitive hearing devices that only need to be pointed toward your house to pick up your conversation. It is one thing to lock up your letters and keep the key. It is quite another to protect your e-mail when hackers can retrieve documents from mainframes no matter how hard someone may try to secure them.

Yet this is just the problem modern pedestrians face because of new modes of transportation. The means of causing harm have changed, but the kind of harm is the same. It is harder being a pedestrian these days, and it is harder protecting one's privacy. But, on this view, someone invading your privacy through modern technological devices is still invading your privacy, and the concept of privacy has not changed, despite the increasing number of ways in which privacy can be invaded and the greater harm that can occur.

However one parses out this view, it will have at least two features:

1. The harm is caused by an invasion, some act (or omission) on the part of whoever is at fault.

If there is intrusion, some person or set of persons has intruded. If there is disclosure, some person or set of persons has disclosed. In each case, we can fault some person or set of persons. It is that person or set of persons who create the harm by telling something they were told in strict confidence, by intruding into a space in which they do not belong, by penetrating, in some way, the envelope of privacy each of us normally carries with us.

The implication of this feature is that the person whose privacy is being invaded is not a voluntary party to the proceedings-has not invited in the person who 
intrudes, has not given those in on the secret permission to tell anyone else. This is not to deny that the person may have done something voluntarily that might encourage an invasion. Leaving one's bedroom door closed but unlocked when there are small children in the house is an invitation to an invasion by small children-although it ought not to be an invitation to older children, who should have learned that a closed door means that knocking first is required, or to other adults who ought to understand what a closed bedroom door could signify. But what marks out invasions of privacy as harms is that they are invasions, something done to someone without that person's consent.

A second feature of this understanding of the concept of privacy thus follows from the first:

\section{Invasions of privacy are intrinsically harmful.}

Suppose a parent reads a child's diary, a diary the child thought was private, and then discloses what is read to others in the family. That disclosure may cause further harms as, for instance, information about how the child really feels about a sibling makes its way through the family. Or the child may come to see the invasion itself as an instance of a controlling parent, and that perception may reverberate through what ought to be a loving relationship and forever alter its cast, precluding the kind of relation that ought to define a parent and a child. Such harms may have an extended life, that is, and so the extrinsic harms of such invasions may be great. Yet their occurrence is a causal contingency, not guaranteed by the intrinsic harm guaranteed to occur through the invasion itself.

However we understand the concept of privacy invoked by the four traditional privacy torts, we shall need to leave room for two important features. First, invading it is an event, among other events in the world, and, just as with other events, it has effects, which may be good, bad, or indifferent, depending upon the circumstances. A well-conceived minor disclosure may have disastrous consequences just as a major intrinsic harm may have no untoward effects at all. It will all depend upon the causal circumstances. Second, whatever the effects, the invasion itself is harmful, and our understanding of the concept must account for why it is intrinsically harmful to invade someone's privacy-harmful even if there are no untoward effects and even if the harm of the invasion itself is relatively minor. What values are invoked when we decry invasions of privacy?

Unfortunately, as Prosser (1960) claimed, no single value or set of values seems to explain how it is that four privacy torts have developed in the law, what underlying values are implicated in these harms. The concept of privacy is complex, with a complicated legal and philosophical history. One feature of that history is the attempt to find a common core for the concept, one value to which all invasions of privacy are reducible. It might thus be construed as the taking of property, rendering all the torts as appropriations (but see Thomson, 1975). Gerety (1977, pp. $263 \mathrm{ff}$.) construed it as intrusion so that disclosure, for example, becomes a way of intruding upon someone's privacy, and Fried (1968, p. 475) construed it as disclosure so that intrusion becomes a form of disclosure. ${ }^{1}$

These reductions each invoke different values. On Fried's (1968) analysis, what is most of value is freedom to control information about ourselves. That control gives us power to regulate our relations with others, creating intimates of some, by telling them the details of our lives, making friends of others, by giving them much but not letting them into the circle of, intimacy, making strangers of many, by giving them no information about ourselves. The value is freedom, in particular freedom of expression, and its loss means an erosion of the relations that depend upon it-friendship, for instance.

On Gerety's (1977) analysis, what is most of value is respect for individuals. When someone comes into the room where you are making love, staying and staring rather than apologizing and leaving, you are being treated like objects. There is a subsequent loss of freedom of expression, obviously, because the person intruding sees what you would presumably not have broadcast about, but the main harm, and so the main value invoked, is that you are being viewed, without your permission-as we might view any objects in the world. We can get a sense of what Gerety (1977) meant to invoke by supposing the person saying to some strangers in tow, some children, for instance, "Here's what I mean! This is what it is like to make love." We would have lost control over information about ourselves, obviously, but in a way to demean us. We would be treated as curiosities, objects to be examined, not persons to be respected.

If we construe our privacy as a property right, someone who invades it harms us in the way we are harmed when a burglar breaks into our house and steals a stereo. The envelope of privacy really is, on this view, a strange kind of object that, when invaded, is taken from us. Information in our diaries is our property just as what is seen in an intrusion must belong to us.

It is not obvious how to mediate these disputes about what is fundamental to the concept of privacy. Each reduction seems to resonate with some feature of the torts not taken as primary. We do lose control over information, for instance, when someone intrudes on our privacy or appropriates our image or name. But the reduction of all the kinds of harm to one particular kind seems driven as much by an a priori ranking of values as by features of the harms themselves, and, in any event, no consensus has emerged about what value is most important, which one is always invoked.

'False light has escaped as a candidate for anything. No one has chosen to reduce all privacy torts to false light, and each has rejected false light as worth reducing. The problem is that it seems not to be an invasion of privacy at all. After all, how can one invade someone's privacy by saying something false about it? False light looks like bungled disclosure and not a separate kind of harm. I think this understanding mistaken (Robison, in press), but, I would argue, any difference a proper understanding of false light makes to our understanding of the concept of privacy will be captured by a proper understanding of the other privacy torts. 
It is a mistake, therefore, to suppose that a single concept of privacy captures the old-fashioned ways of invading privacy and so can be used to clarify how privacy is invaded in new ways. Yet there seems to be little difference between reading e-mail and reading mail, and so it seems easy to graft new ways of invading privacy onto some of the old ways. But the ease with which we can do this is part of the problem. It prevents us from seeing just how radically different is the concept of privacy being invoked by the use of new technologies.

Indeed, we can get a sense of the change by considering in more detail appropriation- of the old-fashioned sort. The standard cases are ones in which a person's name or visage are used for commercial purposes without permission and so without compensation. The oldest is one in which a young socialite in New York City had her photograph taken for her coming out and later saw her picture on sacks of flour with the logo, "The Flour of the Family." She sued and lost, and that led to the New York legislature's introducing the first law protecting a right to privacy-a right not to have one's name or visage used without permission (Prosser, 1960, p. 385).

This hardly seems a privacy right. We can imagine primitive tribes who might think a person's name so tied to his or her identity that to let it out would be to court disaster. The members of the tribe might use false names in public. We can readily imagine cultures in which one's visage is a private matter, not to be viewed by the public at large. A woman's face in some Islamic cultures is private in this way. But in our culture both names and faces are public, and though we can see why one might be thought to have a property right in both, a right requiring compensation when the name or visage is used by others-otherwise models would make no money-it is hard to see how using someone's name or visage without permission is an invasion of privacy.

However, appropriation is limited neither to one's name or image nor to someone gaining a commercial advantage. That is, it is not obviously a property right. Consider the following case. A man pretending to be Vidal, the famous fashion photographer, would approach attractive women in bars with the following line:

Excuse me, you are a very attractive woman. The bone structure in your face is particularly fine. My name is Vidal. I'm a fashion photographer. You may have seen my work in Vogue. I would like to take some photographs of you to see if you would work as a model for me. I can't guarantee anything, I must say. Some beautiful women simply do not photograph well. But if you're willing, I would be interested.

He would then take those willing to his room, pretend to take photographs, seduce them if he could, and, in some cases, "borrow" money.

Vidal found out about this because women called him to find out what had happened with the photographs. The man was caught when Vidal went public, the story got published on the front pages of many newspapers, and the same day it appeared, the man approached a woman at a bar in Washington, DC. She had read. the article and agreed to meet him in his room after visiting the powder room. Instead she called the police, who arrested him ("The Phony Photographer," 1997).
What is the harm to Vidal? No one intruded on him, and no one dinclowed anything he wished to keep private. His impersonator only made use of publicly available information. Indeed, the scam would not have worked if Vidal were not a public figure and the information the con man used-such as that Vidal's photographs appear in Vogue-were not well-known. So appropriation in the old-fashioned sense is not reducible to either disclosure or intrusion. But it is also not just a loss of property, for someone else's commercial advantage. Vidal's identity was appropriated, not any property of his, and it was appropriated for noncommercial purposes. ${ }^{2}$

If Vidal was harmed, his privacy-in any old-fashioned sense-was not invaded. Consider the two conditions we drew out thinking of the concept of privacy as an envelope which can be penetrated by others. The first does not obtain. Only public information was used in the impersonation, and because we drew the second feature-that invasions are intrinsically harmful-out of the first, we would need a new explanation for why the impersonation is intrinsically harmful, if it is. I argue that invasions of privacy are intrinsically harmful because the person whose privacy is being invaded is not a voluntary party to the proceedings. If you invite a crowd in to watch you make love, you have no right to complain that your privacy is invaded. Whether or not Vidal wants people to know that he is a famous photographer and publishes in Vogue, he has no control over who has that information and who does not. It is not just a fact about Vidal that he publishes in Vogue, but a fact about Vogue as well, knowable by any who look at the magazine. But if the information the impersonator used was not the result of any invasion of Vidal's privacy, how can his privacy be at issue? What values do we decry when we object to what happened to Vidal?

We can begin to tease out the beginnings of an answer, and a clue to understanding how the concept of privacy is changing, by examining a relatively new phenomenon that is occurring with increasing frequency. Consider the following:

In December 1993, Richard F. Johnson, the owner of a Carlsbad, California gas station, received startling news when he tried to refinance the loan on his Southern California home: Credit card companies reported that he owed tens of thousands of dollars for charges and cash advances in the Chicago area.

Not only did Johnson know nothing about the debts, he hadn't even lived in Chicago for more than 15 years.

As it turned out, a man named Frank Evans had been using Johnson's name for 18 years, always paying enough on the cards to satisfy the credit card companies and keeping "the debts low enough so that the real Johnson didn't suffer any adverse

\footnotetext{
What the Vidal case makes clear is just how metaphorical is the claim that our image and our num can be appropriated, for it is only in a metaphorical sense that we can say that Vidal's identity oror hit image or his name-belongs to him in the way his diary and personal phone list do.
} 
consequences." Evans declared bankruptcy, was arrested for stealing a $\$ 10$ cap, and purchased the handgun with which he committed suicide-all in Johnson's name. Johnson had to spend thousands to determine what went wrong with his credit, and his credit record is still problematic because the bankruptcy continues to turn up ('Imposter's Luck. ... " 1997).

Johnson's experience is becoming increasingly common as individuals discover that others, whom they have never met, have taken over their identities for financial advantage. As the Vidal case makes clear, someone may appropriate someone else's identity for all sorts of reasons, not just financial gain, but the usual scenario is that someone takes out credit cards in someone else's name, nuns up huge bills within 30 to 60 days, and then trashes the cards, leaving the person whose identity was taken with the subsequent financial mess.

As in the Vidal case, what is taken is someone's identity, and it is taken by means of information over which the person whose identity is taken has little or no control. The situation is not at all like what happens in disclosure or intrusion. When you walk in on a couple making love, you thereby gain information about them. The intrusion is the source of the information, and the two are harmed intrinsically, and may be harmed extrinsically, by your intruding and having that information. But if they invite you in, they are allowing that information beyond the bedroom door and then they lose control over it.

The transition from our old way of understanding privacy to a newly emerging concept is marked by the way in which we are effectively inviting people into our lives. We do this, often unwittingly, by voluntarily giving up information about ourselves. We have no choice if we are to live in the modem age.

When you go to see your physician, for instance, you voluntarily give up a great deal of intimate information about yourself. You want your physician to know the state of your health, and you want this information collected together, in some convenient way, so that when it is needed for your well-being it is readily available. You might think that you are giving that information only to your physician and to those who work in the physician's office. It would thus surprise you that to save money, some physicians now hire out the writing up of their notes to individuals who work at home, are untrained in medical practice, and are often not required by law to keep confidential whatever they record. So the physician's notes are being read, because they are being transcribed, by some third party-who might be a neighbor, a friend, or a relative making extra money in his or her spare time. ${ }^{3}$

It should not surprise you to discover that the substance of your physician's report on your health is forwarded to your insurance company, but it might surprise

${ }^{3}$ Because the individual is untrained in medical practice and is transcribing the rough notes of the physician, taken during an examination, mistakes can readily get into the data, with the potential for serious mistakes for any who must rely on those data. So, besides being harmed by having private medical information available to other than your health-care providers, you risk harm by health-care providers relying on your medical records. you that "most managed care companies and insurers require that, in order to get coverage, patients sign broad releases-often allowing their records to be given to interstate entities that are not covered by. . . laws" in states prohibiting disclosure except when authorized by the patient (Lewin, 1996, p. D20). So your medical history and medical problems, voluntarily given up, for good reason, may be open to a wide variety of companies and individuals with no particular interest in your health care or in maintaining your privacy. In fact, the market in medical databases is growing. Companies concerned to cut their health-care costs, for instance, and concerned not to hire anyone with potential psychiatric problems, profit from using such databases when hiring.

The problem is quite generalizable. Every time you telephone in a catalog order, for example, you voluntarily give up to the catalog company and to the credit card company information about what you have purchased and information about you necessary for the purchase. Every time you provide such information, you help build a detailed profile of yourself, a profile that before too long displays pattems of behavior of which you may not be aware and with more details than you would be able to recall. ${ }^{4}$ You become a pattern of information.

The path from the old concept of privacy to a new can be nicely marked out by using the image with which Karr (1995) began The Liar's Club:

We have our secrets and our needs to confess. We may remember how, in childhood, adults were able at first to look right through us, and into us, and what an accomplishment it was when we, in fear and trembling, could tell our first lie, and make, for ourselves, the discovery that we are irredeemably alone in certain respects, and know that within the territory of ourselves, there can be only our footprints. (p. ii)

We can lead others into the territory of ourselves, showing them the secret pathways to our soul, or exclude them by misleading them or not saying anything at all. We do this by controlling what about ourselves we let others have. When someone intrudes on us or discloses to others information about us that we would have kept private, as a physician does who hires someone to transcribe notes, we lose that control and are presented to others as we would not necessarily have chosen to be presented. Others have a map, however partial, to "the territory of ourselves," and to those with a map, we become objects, something to be examined and manipulated.

We are, so far, still within the confines of the our old understanding of the concept of privacy as an envelope of protection, but we move beyond that when

'There are databases everywhere. When MTV executives were touring Blockbuster's facilities prior to its acquisition, they were so intrigued by Biockbuster's database on its customers that they started "shouting out the names of famous people and asking what movies they had rented" (Shapiro, 1997, p. A8).

This is a quotation from Laing's The Divided Self, p. 37 
we give out information about us that is gathered together in an ever-expanding bundle so that those with access to it can know more about us than we can remember or are even aware of. For then not only have we lost control over information about ourselves, we have begun to lose control over ourselves-over, at a minimum, what happens to us as in the odd sense of "us" that Mr. Johnson finds himself in when he is thought to have a bad credit record. We have become objects to be appropriated and are no longer persons whose privacy has been invaded.

The problems are all there in the Vidal example. The impostor wanted to seduce women, and how better able to do it than to pretend to be Vidal who, with no ulterior motives, could readily approach women as the impostor did and have a greater chance of success. But in taking over Vidal's identity, the impostor treated Vidal as an object to be manipulated to his own ends. One effect is to constrain Vidal's freedom of action. Vidal is no longer as free as he had been to approach women he finds attractive, introduce himself, and suggest that they may wish to be photographed. He might not wish to importune women in that way, finding such behavior boorish, and another harm is that his reputation is stained, at least for those who think that Vidal would behave that way. By appropriating Vidal's identity, the impostor limited Vidal's freedom of action and demeaned him, making Vidal appear responsible for matters he might find offensive.

But beneath these harms is the harm of appropriation, of treating someone's identity as though it were an object, an overcoat, that one could put on, use for one's own purposes, and then discard. The harms are both extrinsic and intrinsic. The extrinsic harms are those that come from how others now perceive the real owner, who must bear the burdens created by the impostor's impersonation. In Johnson's case this means great expense and a lingering suspicion that he is not completely trustworthy financially. In Vidal's case this means at least a loss of freedom. But it is in the intrinsic harm that we find the core value. For to treat our identities as though they were objects to be manipulated is to deny our status as autonomous moral agents, worthy of respect because we are autonomous and not objects.

What we find emerging is a way of understanding the concept of privacy that reconciles that divergent set of values that marked our understanding of the old privacy torts. There is something appropriated, but it is our identity, not our property. It is because it is appropriated, treated as property, that we are treated as objects and so harmed. It can be appropriated because, as happens with disclosure, we have lost control over information about ourselves and thus over how we represent ourselves to others. We are, to that extent, like objects, subject to the whims of others and not to our own free will, because those with information about us may disclose what they wish when they wish to whom they wish, manipulating our image for whatever ends they wish. There is a loss of liberty and following on that a loss of dignity and respect. As with intrusion, we are treated like objects and so demeaned.

So privacy is not a minor value. It connects up with what some would argue is the most important moral value we can have, namely, our being moral agents, individuals with the power and the right to control our place in the world at large, by choosing what to do and what not to do, what to let others know about ourselves and what to keep to ourselves. One way we protect ourselves, and protect our dignity as autonomous moral agents, is to control how we present ourselves to the world. When we are treated like packets of information, to be appropriated, we have lost control over who we are-how we are perceived in society. To have others appropriate us is to fail to respect us and so fail to respect our choices about how we would like to present ourselves to the world. Anything that puts privacy at risk thus puts at risk our sense of ourselves as moral agents.

\section{REFERENCES}

Alderman, E., \& Kennedy, C. (1995). The right to privacy. New York: Knopf. Fried, C. (1968). Privacy. The Yale Law Journal, 77, 475-493.

Gerety, T. (1977). Redefining privacy. Harvard Civil Rights-Civil Liberties Law Review, 12(2), 233-295.

Imposter's luck, and credit, finally run out. (1997, January 1). Democrat \& Chronicle, pp. A1-A2.

Karn, M. (1995). The liars" club: A memoir. New York: Penguin.

Laing, R. D. (1959). The divided self. London: Penquin Books.

Lewin, T. (1996, June 22). Questions of privacy roil arena of psychotherapy. New York Times, pp. A1, D20.

The phony photographer. (1997, September 19). Newsweek, p. 43.

Prosser, W. L. (1960). Privacy. California Law Review, 48(3), 383-423.

Robison, W. (in press). False light. In anthology on liberty, untitled. Lawrence: University of Kansas Press.

Shapiro. E. (1997, February 21). How Viacom's deal for Blockbuster chain went sour so fast. Wall Street Journal, pp. A1, A8.

Thomson, J. J. (1975). The right to privacy. Philosophy and Public Affairs, 4, 295-314.

Warren, S., \& Brandeis, L. (1890). The right to privacy. Harvard Law Review, 4, 75-103. 